\title{
The Leishmania amazonensis TRF (TTAGGG repeat-binding factor) homologue binds and co-localizes with telomeres
}

\author{
Marcelo S da Silva', Arina M Perez¹, Rita de Cássia V da Silveira'1, Camila E de Moraes', Jair L Siqueira-Neto², \\ Lucio H Freitas-Junior² and Maria Isabel N Cano*1
}

\begin{abstract}
Background: Telomeres are specialized structures at the end of chromosomes essential for maintaining genome stability and cell viability. The importance of telomeric proteins for telomere maintenance has increased our interest in the identification of homologues within the genus Leishmania. The mammalian TRF1 and TRF2 proteins, for example, bind double-stranded telomeres via a Myb-like DNA-binding domain and are involved with telomere length regulation and chromosome end protection. In addition, TRF2 can modulate the activity of several enzymes and influence the conformation of telomeric DNA. In this work, we identified and characterized a Leishmania protein (LaTRF) homologous to both mammalian TRF1 and TRF2.

Results: LaTRF was cloned using a PCR-based strategy. ClustalW and bl2seq sequence analysis showed that LaTRF shared sequence identity with the Trypanosoma brucei TRF (TbTRF) protein and had the same degree of sequence similarities with the dimerization (TRFH) and the canonical DNA-binding Myb-like domains of both mammalian TRFs. LaTRF was predicted to be an $82.5 \mathrm{kDa}$ protein, indicating that it is double the size of the trypanosome TRF homologues. Western blot and indirect immunofluorescence combined with fluorescence in situ hybridization showed that LaTRF, similarly to hTRF2, is a nuclear protein that also associates with parasite telomeres. Native and full length LaTRF and a mutant bearing the putative Myb-like domain expressed in bacteria bound double-stranded telomeric DNA in vitro. Chromatin immunoprecipitation showed that LaTRF interacted specifically with telomeres in vivo.

Conclusion: The nuclear localization of LaTRF, its association and co-localization with parasite telomeres and its high identity with TbTRF protein, support the hypothesis that LaTRF is a Leishmania telomeric protein.
\end{abstract}

\section{Background}

More than 20 Leishmania species are pathogenic to humans and cause leishmaniasis of differing severity. Leishmania amazonensis (Trypanosomatidae), the parasite studied in this work, is common in Brazil and causes a wide spectrum of clinical leishmaniasis [1]. The parasite can cause opportunistic infections in HIV/AIDS patients and co-infections have been reported in 34 countries. There are no adequate methods for controlling leishmaniasis and current available treatments are inefficient $[2,3]$. Consequently, most of the ongoing research for new

\footnotetext{
*Correspondence: micano@ibb.unesp.br

1 Telomeres Laboratory, Department of Genetics, Biosciences Institute, Universidade Estadual Paulista Júlio de Mesquita Filho, UNESP, Botucatu, SP, Brazil

Full list of author information is available at the end of the article
}

drugs to combat the disease is based on post-genomic approaches [4].

Telomeres are specialized structures at the end of chromosomes and consist of stretches of repetitive DNA (5'TTAGGG-3' in vertebrates and trypanosomatids) and associated proteins [5]. Telomeres are essential for maintaining genome stability and cell viability, with dysfunctional telomeres triggering a classic DNA-damage response that enables double-strand breaks and cell cycle arrest [6].

There are three classes of telomeric proteins, viz., proteins that bind specifically to single-stranded G-rich DNA, proteins that bind to double-stranded DNA and proteins that interact with telomeric factors. Other nontelomeric proteins, such as the DNA repair proteins

( 2010 da Silva et al; licensee BioMed Central Ltd. This is an Open Access article distributed under the terms of the Creative Commons BFoMed Central Attribution License (http://creativecommons.org/licenses/by/2.0), which permits unrestricted use, distribution, and reproduction in any medium, provided the original work is properly cited. 
Mre11 and Rad51, also play important roles at telomeres $[7,8]$. In mammals and yeast, telomeric proteins are organized in high order protein complexes known as shelterin or telosome that cap chromosome ends and protect them from fusion or degradation by DNA-repair processes $[9,10,7]$. These complexes, which are abundant at chromosome ends but do not accumulate elsewhere, are present at telomeres throughout the cell cycle and their action is limited to telomeres $[7,8]$. Shelterin/telosome proteins include members or functional homologues of the TRF (TTAGGG repeat-binding factor) or telobox protein family, such as TRF1 and TRF2 from mammals [11] and Tebp1 [12], Taz1 [13] and Tbf1 [14] from yeast. All of these proteins bind double-strand telomeres via a Myblike DNA-binding domain, which is one of the features that characterize proteins that preferentially bind doublestranded telomeric DNA [15-17].

In humans, TRF1 may control the length of telomeric repeats through various mechanisms. For example, TRF1 can control telomerase access through its interaction with TIN2, PTOP/PIP1 and the single-stranded telomeric DNA-binding protein POT1. TRF1 may also regulates telomerase activity by interacting with PINX1, a natural telomerase inhibitor. In comparison, TRF2 is involved in many functions, including the assembly of the terminal tloop, negative telomere length regulation and chromosome end protection $[18,11,16]$. The shelterin complex is anchored along the length of telomeres by both TRF2 and TRF1 [19], whereas in conjunction with POT1, TRF2 is thought to stimulate WRN and BLM helicases to dissociate unusual structures during telomeric replication [20]. TRF2 also interacts with enzymes that control G-tail formation, the nucleases XPF1-ERCC1, the MRE11-RAD50NBS1 (MRN) complex, the RecQ helicase WRN and the 5 ' exonuclease Apollo [8]. Loss of TRF2 leads to NHEJmediated chromosome end-fusion and the accumulation of factors that form the so-called telomere dysfunctioninduced foci (TIFs) $[21,22]$. Thus, TRF2 can modulate the activity of several enzymes and influence the conformation of telomeric DNA.

Only a few telomeric proteins that bind the doublestranded form of telomeric DNA have been described in Leishmania and in their trypanosome counterparts $[17,23]$. Homologues of human TRF have been found in the genomes of T. brucei, T. cruzi and L. major based on sequence similarities to the C-terminal Myb-like DNA binding domain. For example, the T. brucei TRF2 homologue known as TbTRF shares a similar telomere endprotection function with vertebrate TRF2 [24].

\section{Results and Discussion}

Characterization of the putative $L$. amazonensis TRF gene homologue

Using data mining via the OmniBLAST server we searched the whole L. major genome database http:// www.ebi.ac.uk/parasites/leish.html for a putative sequence that shared similarities with the vertebrate TRF1 and TRF2 proteins. For this search, we used the most conserved part of both human proteins, the C-terminal fragment containing the Myb-like DNA binding domain. The search returned a single sequence (GenBank acc. no. XP 001682531.1) that encoded a hypothetical protein (GenBank acc. no. Q4QDR7, GeneDB_Lmajor LmjF18.1250), the C-terminus of which shared $~ 30 \%$ identity and $50-55 \%$ similarity with the vertebrate TRF Myb-like domain, according to the blast 2 sequence analysis (Table 1).

Based on the L. major sequence, primers were designed for PCR amplification of the entire homologous sequence from L. amazonensis with genomic DNA as the template. PCR products of 2,931 bp were cloned into the vector pCR2.1 and both insert strands were sequenced (data not shown). The deduced polypeptide sequence of 796 amino acid residues contained a putative $\mathrm{C}$-terminal Myb-like DNA binding domain between residues 684-733, according to psi-blast (Fig 1 - top). The LaTRF gene (GenBank acc. no. EF559263) shared high sequence identity and similarity to the putative L. major TRF, and to hypothetical L. infantum and L. braziliensis TRFs (Table 1). The sequence conservation between LaTRF and the trypanosome TbTRF and the putative TcTRF homologues decreased to $35-45 \%$ identity (Table 1 ), consistent with the known evolutionary relationships among these organisms. The Leishmania TRF homologues encode the largest TRF protein $(\sim 82.5 \mathrm{kDa})$ described so far. The fact that the Leishmania proteins showed much greater homology with each other than with other protozoan proteins and that they are the largest TRF described so far resembles the situation for Leishmania telomerase protein [25].

In addition, like TbTRF, LaTRF shared sequence similarities with the canonical Myb-like domain and with the TRFH dimerization domain of human TRF1 and TRF2 (Fig 1-bottom and Table 1), but no sequence similarities were found with any other telobox protein (data not shown). Together, these results indicate that although LaTRF shares high sequence similarity with TbTRF, probably because the two species are phylogenetically related [26], further studies are required to confer any functions to the Leishmania TRF homologue identified here.

\section{LaTRF is a nuclear protein that co-localizes with $\mathrm{L}$. amazonensis telomeres}

In exponentially growing L. amazonensis promastigotes, LaTRF was detected only in nuclear protein extracts. A single $\sim 82.5 \mathrm{kDa}$ protein band was detected using antiLaTRF serum (Fig 2 - top panel: lane 1). No protein was detected in cytoplasmic and total protein extracts (Fig 2 top panel: lanes 2 and 3), indicating that LaTRF is a nuclear protein with very low intracellular abundance. As 
Table 1: Pairwise analysis of amino acid sequence alignments from TRF homologues based on bl2seq sequences (proteinprotein BLAST)

\begin{tabular}{|c|c|c|c|c|c|c|}
\hline & \multicolumn{2}{|c|}{ LaTRF (full length) } & \multicolumn{2}{|c|}{ LaTRFTRFHdomain } & \multicolumn{2}{|c|}{ LaTRFMybdomain } \\
\hline & \%Identity & \%Similarity & \%Identity & $\%$ Similarity & \%Identity & \%Similarity \\
\hline LmTRF & 99 & 99 & 100 & 100 & 100 & 100 \\
\hline LiTRF & 88 & 91 & 85 & 89 & 98 & 100 \\
\hline LbTRF & 65 & 71 & 60 & 70 & 96 & 100 \\
\hline TcTRF & 45 & 59 & 38 & 54 & 63 & 77 \\
\hline TbTRF & 35 & 53 & 39 & 59 & 54 & 66 \\
\hline hTRF1 & Not significant & Not significant & 16 & 25 & 31 & 54 \\
\hline hTRF2 & Not significant & Not significant & 15 & 30 & 29 & 55 \\
\hline
\end{tabular}

a control, Western blots were revealed with anti-LaRPA-1 serum, which recognizes a $\sim 51.2 \mathrm{kDa}$ telomeric protein band [23] (Fig 2 - bottom panel: lane 1) and also its phosphorylated forms (Fig 2 - bottom panel: lane 2; da Silveira \& Cano, unpublished data).

We also developed an immunofluorescence assay combined with FISH, using anti-LaTRF serum and a PNAtelomere probe specific for TTAGGG repeats. As shown in Fig 3 (panels p1-4, merged images a and b), LaTRF is a nuclear protein that partially co-localizes with parasites telomeres, since some of the LaTRF signal coincided with telomeric foci and some did not (Fig 3, panels p1-4). In most cells, LaTRF appears as a diffuse signal spread all over the nucleoplasm and only in some cases it forms large punctuated foci, which seems to co-localize with the telomeric DNA (yellow dots in Fig 3, panels p2 and p4). Similarly, in humans, the hTRF2 protein also appears in the form of punctuate foci that does not completely associate with telomeres [18], which is in agreement with other cellular functions played by this protein $[27,28]$. In contrast, the T. brucei TRF protein (TbTRF) appears to co-localize with most telomeres at all stages of the cell

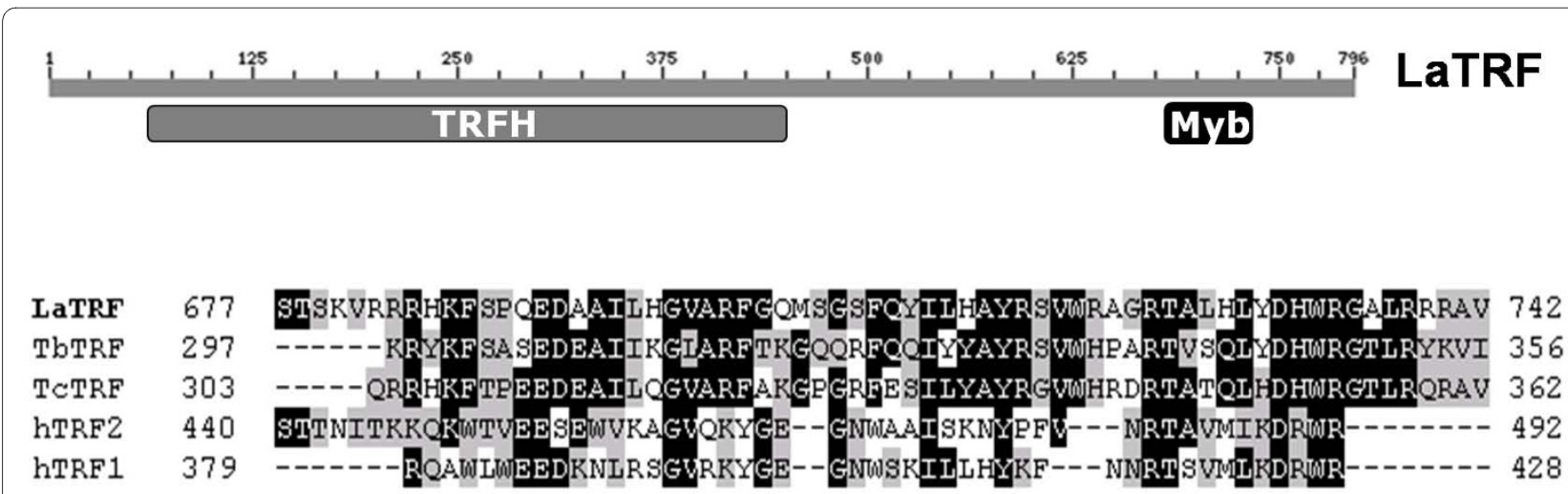

Figure 1 LaTRF is a homologue of mammalian and T. brucei telomeric TRFs. (top) Position of the TRFH and Myb domains in LaTRF, according to rpsblast and bl2seq sequence analysis with T. brucei TRF. (bottom) ClustalW multiple alignment of the Myb-like DNA binding domains of human (hTRF2 and hTRF1), L. amazonensis (LaTRF), T. brucei (TbTRF) and T. cruzi (TcTRF) TRFs. 


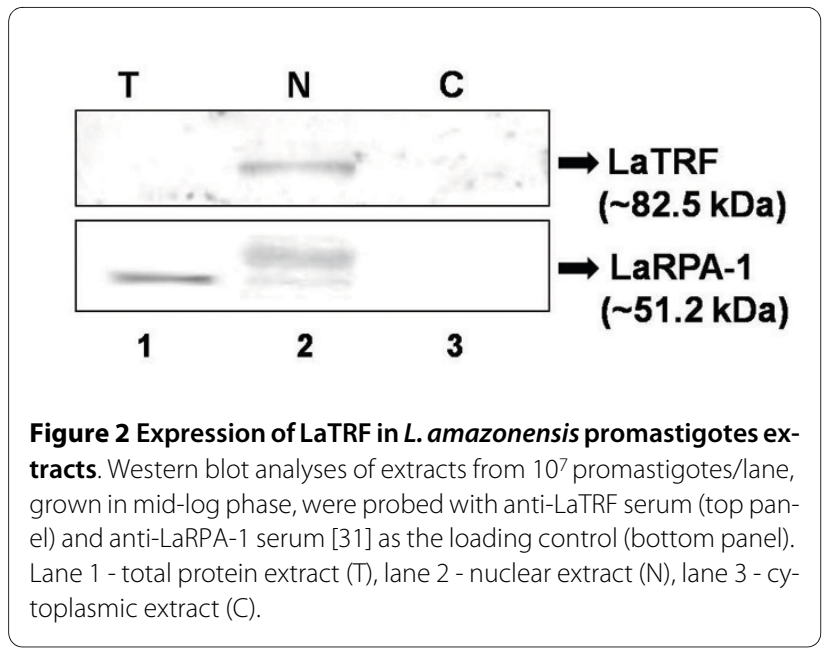

cycle in both bloodstream and procyclic forms [24]. Whether LaTRF also has other cellular roles or if its association with telomeres occurs in a cell cycle dependent manner is not clear at this stage.

\section{LaTRF interacts in vitro and in vivo with $L$. amazonensis telomeres using a Myb-like DNA binding domain}

EMSA assays were done with renatured protein extracts containing full length LaTRF, the Myb-like DNA binding domain (LaTRFMyb) (Figs 4 and 5, see additional file 1) and with L. amazonensis nuclear extracts (Fig 6), to investigate whether LaTRF, like its vertebrate and trypanosome counterparts $[18,24]$, was able to bind doublestranded telomeric DNA in vitro.

The full-length recombinant protein and its deletion mutant were expressed in very low amounts and in non-

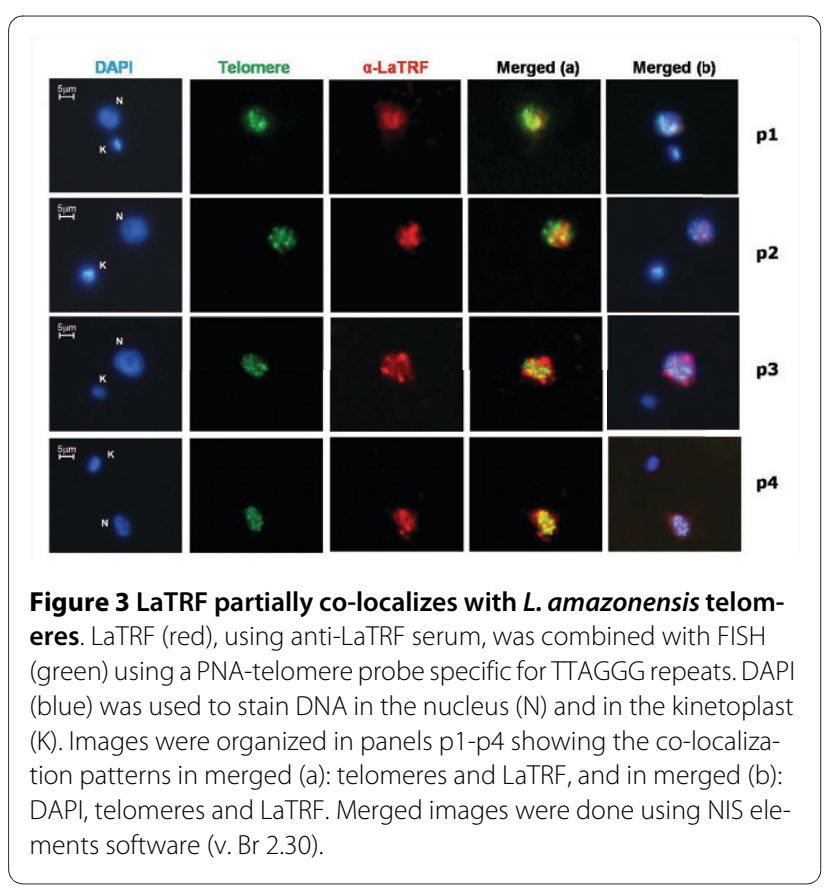

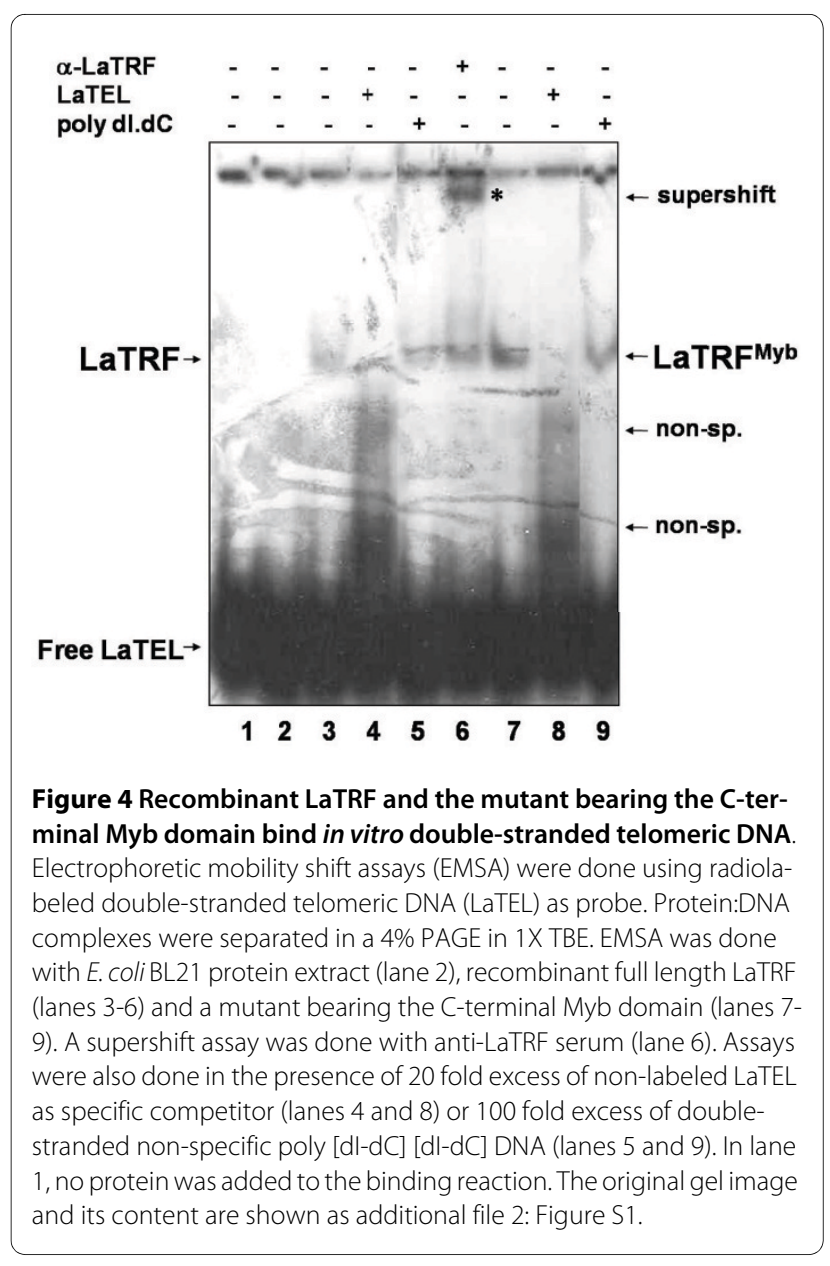

soluble form in the E. coli system (data not shown) making their purification by conventional chromatography very difficult. Therefore, protein expression was checked by Western blot using anti-LaTRF serum and anti-His tag monoclonal antibody (data not shown).

As shown in Fig 4, recombinant full length LaTRF and the mutant bearing only the $\mathrm{C}$-terminal Myb-domain were able to bind specifically the double-stranded telomeric DNA (LaTEL). Competition assays showed that the complexes formed by both recombinant proteins were completely abolished in the presence of excess unlabeled LaTEL and that there was no competition for binding when excess of non-specific poly [dI-dC] [dI-dC] doublestranded DNA was used (Fig 4, lanes 4, 5, 8 and 9). Supershift assay with anti-LaTRF serum, which recognizes a Nterminal epitope in the protein, confirmed that full length LaTRF forms a robust complex with labeled LaTEL (Fig 4, lane 6), possibly because the binding of anti-LaTRF stabilized the LaTRF-LaTEL complex, blocking the action of other non-specific binding activity in the extract. When competitors were added to the supershift reactions with anti-LaTRF serum, the binding specificity of recombinant LaTRF for LaTEL was confirmed (Fig 5, lanes 2-4). The complex was almost totally abolished in the presence of 


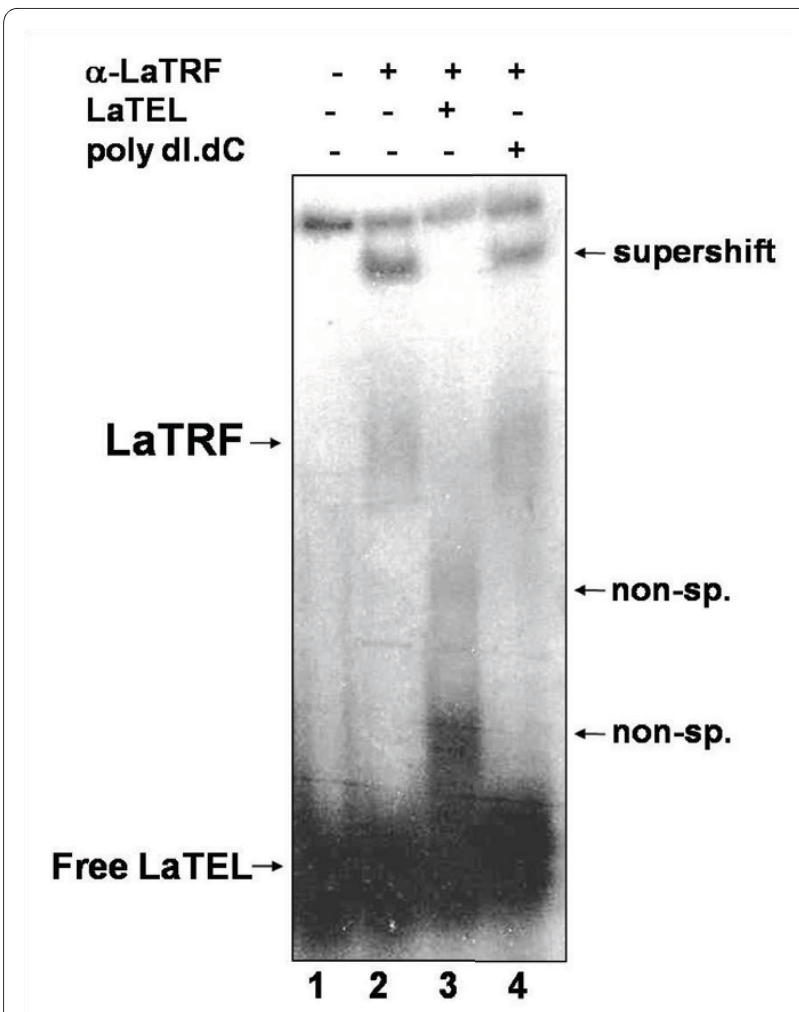

Figure 5 Supershift and competition assays confirm that recombinant full length LaTRF bind in vitro double-stranded telomeric DNA. Electrophoretic mobility shift assays (EMSA) were done using radiolabeled double-stranded telomeric DNA (LaTEL) as probe. Protein:DNA complexes were separated in a 4\% PAGE in 1 XTBE. EMSA was done with recombinant full length LaTRF and anti-LaTRF serum in the absence (lane 2) and in the presence of 20 fold excess of non-labeled LaTEL as specific competitor (lane 3) or 100 fold excess of doublestranded non-specific DNA (poly $[\mathrm{dl}-\mathrm{dC}][\mathrm{dl}-\mathrm{dC}]$ ) as non specific competitor (lane 4). In lane 1 reaction was done in the presence of LaTEL only.

excess unlabeled LaTEL (Fig 5, lane 3) and no competition was detected in the presence of non-specific DNA (Fig 5, lane 4). The results presented above suggest that recombinant LaTRF binds LaTEL potentially via the putative Myb-like DNA binding domain indicating a role for the C-terminal region of LaTRF in mediating sequence-specific binding to telomeric DNA.

Nuclear extracts were obtained from log phase L. amazonensis promastigotes in order to check if native LaTRF was also able to bind double-stranded telomeric DNA (LaTEL) in vitro, (Fig 6). The results showed the presence of LaTRF activity in these extracts, as part of the complex formed with the nuclear proteins and LaTEL (Fig 6, lane 2 ) was supershifted by the anti-LaTRF serum (Fig 6 , lane 6). In addition, competition assays showed that this complex was unaffected by excess of poly [dI-dC] [dI-dC] (Fig 6 , lane 3), used as the non-specific competitor, but it was almost completely abolished in the presence of excess unlabeled LaTEL (Fig 6, lane 4). Supershift experiments

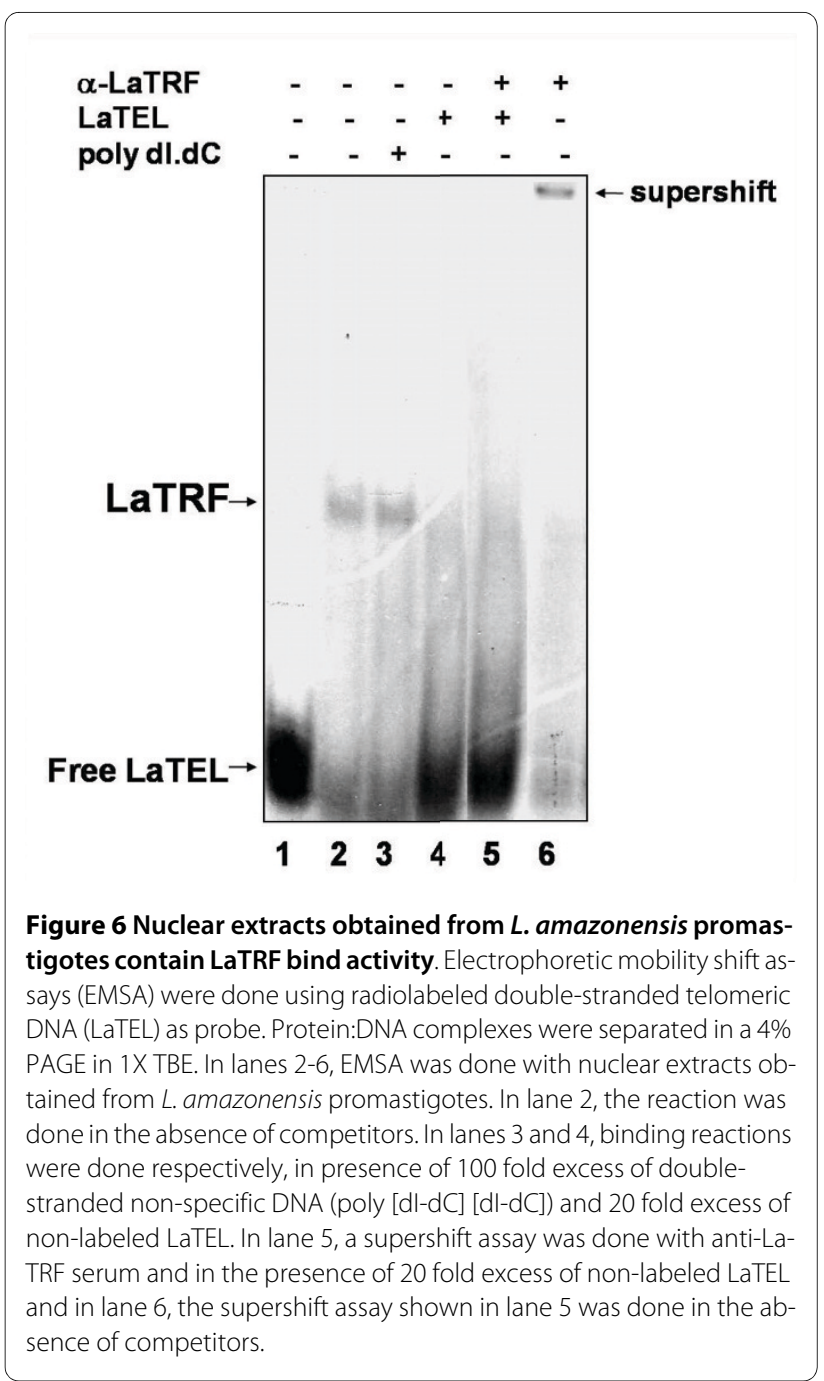

using anti-LaTRF serum were done in the presence of competitor to confirm that LaTRF was actually involved in the formation of the retarded band (Fig 6, lane 5). Note that the retarded shifted band disappeared due to the competition by non-labeled LaTEL. Thus, these results indicate that LaTRF is in part responsible for the binding activity shown in these extracts and is probably a component of the Leishmania telomeric complex.

Chromatin immunoprecipitation experiments also suggested that LaTRF is a telomeric protein. The anti-LaTRF serum immunoprecipitated $L$. amazonensis telomeric DNA (Tel1) in vivo (Fig 7 - left) but did not immunoprecipitate the GT-rich kinetoplast DNA (kDNA) (Fig 7 right). The kDNA control represented by the UMS (universal mini-circle sequence) albeit GT-rich, is very representative of the general base composition of Leishmania genomic DNA. In addition, it is a good control, since we were able to show that it was co-immunoprecipitated by two other Leishmania telomeric protein $[17,23]$. In a previous study, we described LaTBP1, a protein that specifically binds telomeric and GT-rich DNA in Leishmania. 


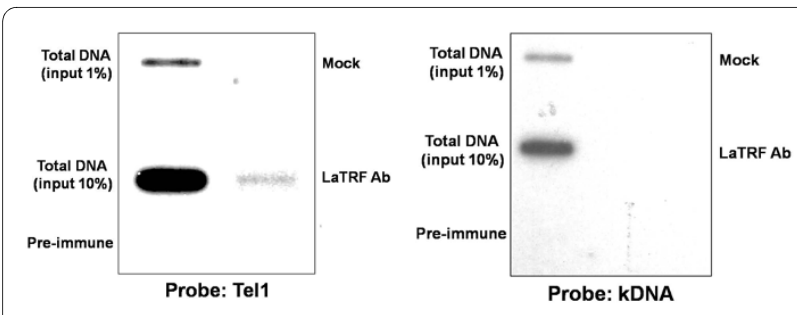

Figure 7 LaTRF interacts with telomeric DNA in vivo. Chromatin immunoprecipitation (CHIP) of mid-log phase promastigotes cells using anti-LaTRF. Control experiments were done with chromatin immunoprecipitated in the presence of pre-immune serum and without serum (mock). Total DNA (input) corresponds to $10 \%$ and $1 \%$ of the amount of DNA in $10^{8}$ cells cross-linked with the chromatin. Slot-blots were hybridized with $5^{\prime}$ end-labeled Tel1 probe (left) and re-hybridized with the kDNA probe (right).

LaTBP1 has a centrally positioned Myb-like DNA binding domain and is most likely a non-telobox protein that is apparently related to the multifunctional yeast RAP1 telomeric protein and TFIIIB B" transcription factor [17]. Together with the putative LaTRF described here, these are the only descriptions of proteins bearing a Myb-like DNA binding domain that interact with double-stranded telomeric DNA in Leishmania.

As mentioned here and elsewhere [26], the huge evolutionary distance between this protozoan and higher eukaryotes presents a barrier when searching for protein homologues in the genomes of these parasites. For example, no TRF1 homologues were found in trypanosomatid genomes but the expression of hTRF1 in procyclic forms of T. brucei caused telomere shortening and cell cycle arrest, probably by displacing an unknown endogenous telomeric factor [29]. RNAi knockdown of TbTRF arrested bloodstream cells in G2/M and most of the procyclic cells in the S phase, and caused shortening of Grich single-stranded telomeric DNA. These findings suggest that TbTRF is probably the unknown endogenous telomeric factor, which resembles the function of mammalian TRF2 at parasite telomeres [24]. The functions of LaTRF at Leishmania telomeres remain to be determined.

\section{Conclusions}

In this report we describe the characterization of the Leishmania TRF homologue and show that it is the largest TRF protein homologue described so far. This protein contains a canonical C-terminal Myb-like DNA binding domain as well as a putative and less conserved TRFH dimerization domain [30]. In addition, LaTRF is expressed exclusively in the nucleus and like its vertebrate and trypanosome counterparts, binds to parasite telomeres in vitro and in vivo. It can also co-localize with parasite telomeres, despite being spread all over the nucleoplasm in most cells, suggesting that LaTRF may play additional cellular roles beyond its possible telomeric function.

\section{Methods}

\section{Parasite cultures}

L. amazonensis promastigotes (MHOM/BR/73/M2269) were grown in M199 medium (Cultilab) supplemented with $10 \%$ fetal calf serum (Cultilab), $25 \mathrm{mM}$ HEPES and 1 $\times$ antibiotic/antimycotic solution (Cultilab) at $28^{\circ} \mathrm{C}$.

\section{Isolation of $\mathrm{L}$. amazonensis genomic DNA and cloning of the LaTRF gene}

Total genomic DNA of L. amazonensis was prepared as previously described [31]. LaTRF was cloned using a PCR-based strategy. Primers were designed based on the putative sequence LM16.2.Contig67 from L. major (GeneDB_Lmajor LmjF18.1250) for amplification of the complete LaTRF open reading frame (ORF) (See additional file 2: Table S1). The PCR product spanning the entire L. amazonensis TRF ORF (2,391 bp) was obtained by using the primers $\mathrm{F} 1$ and $\mathrm{R} 1$ and $1 \mathrm{U}$ of Platinum Taq (Invitrogen) followed by cloning into the $\mathrm{PCR}^{\circ} 2.1$ cloning vector (Invitrogen). The PCR product was sequenced using specific primers and primers from the vector (See additional file 2: Table S1). The primers F1 and R1 contained restriction sites for NdeI and XhoI (See additional file 2: Table S1) to allow further cloning of the gene inframe with a $\mathrm{N}$-terminal $6 \times$ His-tag into plasmid pET$28 \mathrm{a}+$ (Novagen).

Amino acid sequence alignments were done with blastp, bl2seq, cds http://blast.ncbi.nlm.nih.gov/Blast.cgi and ClustalW http://www.ebi.ac.uk/clustalw/ using default parameters. The sequences used for these analyses were: hTRF2 (GenBank acc. no. Q15554), hTRF1 (GenBank acc. no. P54274.2), TbTRF (GenBank acc. no. AY910010), putative LmTRF (TrEMBL acc. no. Q4QDR7, GeneDB_Lmajor LmjF18.1250), TcTRF (GenBank acc. no. XP 819954.1), LiTRF (GenBank acc. no. XP 001464939.1) and LbTRF (GenBank acc. no. XP_001564056.1). The L. amazonensis LaTRF gene sequence was submitted to GenBank and is available under the accession number EF559263.

\section{Construction of an LaTRF deletion mutant (LaTRFMyb)}

To verify the existence of a Myb-like DNA-binding domain at the C-terminus of the protein, a deletion mutant was constructed. The primers F3 and R1 (See additional file 2: Table S1) were used to amplify the deletion mutant $L a T R F^{M y b}$ from genomic DNA, which contained the putative C-terminal Myb-like DNA binding domain. This mutant has approximately 665 bp that span nt 1726-2391. As with full length LaTRF, the LaTRFMyb mutant was cloned into the $\mathrm{PCR}^{\circ} 2.1$ cloning vector 
(Invitrogen), sequenced and subcloned into a pET28a+ expression vector.

\section{Expression of LaTRF and the deletion mutant LaTRFMyb proteins in E. coli}

Full length LaTRF and the deletion mutant $L a T R F^{M y b_{-}}$ cloned into a pET $28 \mathrm{a}+$ vector, were transformed in $E$. coli strain BL21 DE3 RP codon plus cells for expression in the presence of $1 \mathrm{mM}$ IPTG. Both proteins were expressed in low amounts and in non-soluble form, preventing them from being purified by affinity chromatography based on the $6 \times$ His-tag. To overcome this problem, the non-soluble bacterial pellets containing both proteins were solubilized in $7 \mathrm{M}$ urea, sonicated in the presence of $10 \mathrm{U}$ of DNAse I (Sigma) and renatured by dialysis in $50 \mathrm{mM}$ glycine, $\mathrm{pH}$ 8.0. The presence of each protein in the extracts was checked by electrophoresis in 10\% SDS-PAGE followed by Western blot probed with anti-LaTRF serum and with an anti-His tag monoclonal antibody (Novagen).

\section{Preparation of $L$. amazonensis total and nuclear extracts}

Promastigotes in mid-exponential growth were used to obtain both extracts. Nuclear and cytoplasmic extracts were prepared with a Nuclear Extract Kit (Active Motif) adapted for L. amazonensis promastigotes in the presence of phosphatase and protease inhibitors.

Total protein extracts were obtained using RIPA buffer (150 mM Tris- $\mathrm{HCl} \mathrm{pH}$ 7.5, $150 \mathrm{mM} \mathrm{NaCl}, 1 \%$ Triton X100 and $0.1 \%$ SDS) in the presence of $10 \mathrm{U}$ of DNase I and $1 \mathrm{X}$ protease inhibitor cocktail (Calbiochem) and incubated for $15 \mathrm{~min}$ at $4^{\circ} \mathrm{C}$. Cell lysates were homogenized by vortexing at maximum speed (5 bursts of $10 \mathrm{~s}$ each). Extracts were cleared by centrifugation at 9,300 $\times$ g for 8 $\min$ at $4^{\circ} \mathrm{C}$, to separate the total protein (supernatant) from the cellular debris (pellet).

Both extracts were stored at $-80^{\circ} \mathrm{C}$ and their protein concentrations were measured by the Bradford dye-binding assay, using bovine serum albumin as standard.

\section{Western blot analysis}

Different protein extracts obtained from $10^{7}$ parasites were separated by SDS-PAGE on $10 \%$ polyacrylamide gels and transferred to nitrocellulose membranes (BIORAD) in Tris-glycine-methanol at $16^{\circ} \mathrm{C}$. The membranes were probed with rabbit anti-TRF2 serum raised against the synthetic peptide Nt-APAVTTRKRPRSSDSP-Ct (Sigma). The extracts were also probed with anti-LaRPA1 serum as a control [23,32]. In both cases, immunoreactive bands were revealed by using an Amplified Alkaline Phosphatase Immun-Blot Assay Kit, according to the manufacturer's instructions (BIO-RAD).

\section{Indirect immunofluorescence combined with Telomere} PNA FISH (fluorescence in situ hybridization)

This assay was performed using previously described protocols $[32,33]$ with minor modifications. For IF, $10^{6}$ promastigotes cells were washed in 1X PBS and fixed in $1 \%$ formaldehyde for $5 \mathrm{~min}$ at $4{ }^{\circ} \mathrm{C}$. After permeabilization with $0.1 \%$ Triton X-100 (in $1 \mathrm{X}$ PBS) for $10 \mathrm{~min}$ at room temperature, cells were incubated with $0.1 \mathrm{M}$ Glycine (in 1X PBS) and attached to glass coverslips coated with $0.1 \%$ poly-L-Lysine (Sigma). Anti-LaTRF serum was used to detect LaTRF with Alexa Fluor 555-labeled goat anti-rabbit IgG (Invitrogen) as the secondary antibody followed by telomere detection using a Telomere PNA FISH Kit/ FITC (DakoCytomation). VECTASHIELD Mounting Medium with DAPI (Vector Labs) was used as the antifade mounting solution and to stain nuclear and kinetoplast DNA. The images were analyzed with a Nikon 80i fluorescence microscope and captured with a digital camera (Nikon). When necessary, images were superimposed using NIS elements software (v. Br 2.30).

\section{EMSA (electrophoretic mobility shift assay)}

All of the conditions for binding reactions and EMSA, including binding temperature, protein concentrations in the extracts and the double-stranded DNA probe (LaTEL), were standardized in preliminary experiments. LaTEL was constructed by using the $\gamma$ [32P]ATP 5 '-endlabeled oligonucleotides ssTel78G and ssTel78C, as described by Lira et al. [17]. Assays were done by mixing $10 \mu \mathrm{g}$ of renatured bacterial extracts containing full length LaTRF or LaTRF ${ }^{M y b}$ with approximately 2 pmol of labeled probe (LaTEL) in $30 \mu \mathrm{l}$ of EMSA buffer $(20 \mathrm{mM}$ HEPES, $2.5 \mathrm{mM} \mathrm{MgCl}_{2}, 0.1 \mathrm{mM}$ EDTA, $0.1 \mathrm{M} \mathrm{KCl}, 10 \%$ glycerol, $0.5 \mathrm{mM}$ DTT, $\mathrm{pH} 8.0$ ) containing $10 \mathrm{ng}$ of poly [dI-dC] [dI-dC] and 10 ng of poly [dA-dT] [dA-dT]. Total protein extracts of non-transformed E. coli were used as controls. The reactions were incubated for $30 \mathrm{~min}$ at room temperature and loaded onto a non-denaturing $4 \%$ polyacrylamide gel (acrylamide:bis-acrylamide, 19:1, w/ w) in $1 \mathrm{X}$ TBE. After electrophoresis, the gels were exposed to X-ray film.

Binding reactions were also done with crude nuclear extracts obtained from $10^{8}$ parasites $(\sim 2.3 \mu \mathrm{g}$ of total proteins) and $\gamma$ [ $\left.{ }^{32} \mathrm{P}\right]$ ATP labeled LaTEL (2 pmol) in EMSA buffer containing a mixture of $10 \mathrm{ng}$ of poly [dI-dC] [dI$\mathrm{dC}]$ and $10 \mathrm{ng}$ of poly [dA-dT] [dA-dT].

Competition assays to test the binding specificity of proteins in both recombinant and nuclear extracts, were done using 20 fold excess of unlabeled LaTEL (in relation to the labeled probe) as the specific competitor and a 100 fold excess (in relation to the labeled probe) of unlabeled double-stranded DNA poly [dI-dC] [dI-dC] as the non-specific 
competitor. Supershift assays were done using full-length recombinant LaTRF $(10 \mu \mathrm{g})$ or native nuclear extracts from $10^{8}$ parasites in the presence of $\sim 30 \mu \mathrm{g}$ of anti-LaTRF serum in EMSA buffer containing labeled LaTEL as probe and both poly $[\mathrm{dI}-\mathrm{dC}][\mathrm{dI}-\mathrm{dC}]$ and poly $[\mathrm{dA}-\mathrm{dT}][\mathrm{dA}-\mathrm{dT}]$ as above described. These assays were also performed in the presence of 20 fold excess of non-labeled LaTEL and 100 fold excess of poly [dI-dC] [dI-dC] as described above.

\section{Chromatin immunoprecipitation}

Formaldehyde cross-linked chromatin was obtained from promastigote forms of L. amazonensis parasites $\left(0.8 \times 10^{8}\right.$ cells/experiment) as described by Lira et al. [17]. Chromatin was immunoprecipitated with anti-LaTRF serum and DNA was extracted after cross-link reversal. DNA samples were slot-blotted and hybridized with Tel1 and kDNA probes by using a previously established protocol. Aliquots of $1 \%$ and $10 \%$ of total DNA used in each experiment (input) were tested separately. Control assays included immunoprecipitation of chromatin with preimmuneserum (pre-immune) or without serum (mock). The probes used were $5^{\prime}$-end labeled with $\gamma$ ATP [32P]: Tel1 (5'TTAGGG-3') ${ }_{3}$ and kDNA (5'-TTTCGGCTCGGGCGGTGAAAACTGGGGGTTGGTGTAAAAT-3'), according to Lira et al. [17].

\section{Additional material}

Additional file 1 Figure S1. Original and unmanipulated gel image shown in figure 4. EMSA done with radiolabeled double-stranded telomeric DNA (LaTEL) as probe. Protein:DNA complexes were separated in a 4\% PAGE in 1XTBE. In lane 1, no protein was added to the binding reaction. In lane 2, EMSA was done with E. coli BL21 protein extract. In lane 3, EMSA was done with recombinant full length LaTRF. In lane 4, EMSA was done with recombinant full length LaTRF in the presence of 20 fold excess of non-labeled LaTEL as specific competitor. In lane 5, no protein was added to the binding reaction (as in lane 1). In lane 6, EMSA was done with recombinant full length LaTRF in the presence of 100 fold excess of doublestranded non-specific poly [dl-dC] [dl-dC] DNA. In lane 7, EMSA was done with recombinant full length LaTRF in the presence of anti-LaTRF serum (supershift assay). Please check the supershifted complex at the top of the lane. In lane 8, EMSA was done with the mutant recombinant protein bearing the C-terminal Myb domain. In lane 9, EMSA was done with the mutant recombinant protein bearing the $\mathrm{C}$-terminal Myb domain in the presence of 20 fold excess of non-labeled LaTEL. In lane 10, the same experiment shown in lane 9. In lane 11, EMSA was done with the mutant recombinant protein bearing the $\mathrm{C}$-terminal Myb domain in the presence of $100 \mathrm{fold}$ excess of double-stranded non-specific poly [dl-dC] [dl-dC] DNA. In lane 12, the same supershift assay shown in lane 7.

Additional file 2 Table S1 Primers used for PCR amplification and sequencing of the putative $L$. amazonensis TRF gene and the deletion mutant LaTRFMyb. Table containing a list of the primers used for PCR and sequencing assays

\section{Authors' contributions}

MSS performed molecular cloning techniques, designed the deletion mutant, produced recombinant proteins, participated in the sequence alignment analysis, standardized the IF/FISH assays and has been involved in drafting the manuscript. AMP participated in the production of recombinant proteins, performed in vitro binding assays and has also been involved in drafting the manuscript. RCVS and CEM obtained native protein extracts and performed
Western blots and chromatin immunoprecipitation assays. JLSN helped MSS with the cloning strategies, IF/FISH experiments and designed the peptide used to generate anti-LaTRF serum. LHFJ collaborated in outlining some experimental strategies and has been involved in the manuscript revision contributing with important intellectual content. MINC coordinated and designed most of the experiments as well as the strategies used in the manuscript, has mentored MSS, AMP, RCVS and CEM, who have also contributed during discussions of the results. MINC critically read and reviewed the manuscript for its publication. All authors read and approved the final manuscript.

\section{Acknowledgements}

The authors thank Drs. S. Hyslop and J.P. Monteiro for revising the English version of the manuscript. This work was supported by FAPESP (06/58175-7) and CNPq (481850/2008). MSS is supported by an undergraduate studentship from FAPESP. AMP is supported by a doctoral studentship from FAPESP. RCVS and CEM are respectively supported by doctoral and master studentships from CNPq (Brazil).

\section{Author Details}

'Telomeres Laboratory, Department of Genetics, Biosciences Institute, Universidade Estadual Paulista Júlio de Mesquita Filho, UNESP, Botucatu, SP, Brazil and ${ }^{2}$ Center for Neglected Diseases Drug Discovery (CND3), Institut Pasteur Korea, Gyeonggi-do, South Korea

Received: 18 July 2009 Accepted: 7 May 2010

Published: 7 May 2010

\section{References}

1. Barral A, Pedral-Sampaio D, Grimaldi Junior G, Momen H, Mcmahon-Pratt D, Ribeiro de Jesus A, Almeida R, Badaro R, Barral-Netto M, Carvalho EM, Johnson Junior WD: Leishmaniasis in Bahia, Brazil: evidence that Leishmania amazonensis produces a wide spectrum of clinical disease. Am J Trop Med Hyg 1991, 44(5):536-546.

2. Murray HW, Berman JD, Davies CR, Saravia NG: Advance in leishmaniasis. Lancet 2005, 366(9496):1561-1577.

3. Cruz I, Nieto J, Morenot J, Canavate C, Desjeux P, Alvar J: Leishmania /HIV co-infections in the second decade. Indian J Med Res 2006, 123(3):357-388

4. Ouellette M, Olivier M, Sato S, Papadopoulou B: Studies on the parasite Leishmania in the post-genomic era. Med Sci 2003, 19(10):900-909.

5. Cano MIN: Telomere biology of trypanosomatids: more questions than answers. Trends Parasitol 2001, 17(9):425-429.

6. Blackburn $\mathrm{EH}$ : Telomeres and telomerase: their mechanisms of action and the effects of altering their functions. FEBS Lett 2005, 579(4):859-862.

7. de Lange T: Shelterin: the protein complex that shapes and safeguards human telomeres. Genes Dev 2005, 19(18):2100-2110.

8. Longhese MP: DNA damage response at functional and dysfunctional telomeres. Genes Dev 2008, 22(2):125-140.

9. Dimitriev PV, Petrov AV, Dontsova OA: Yeast telosome complex: components and their functions. Biochemistry (Mosc) 2003, 68(7):718-734

10. Liu D, O'Connor MS, Qin J, Songyang Z: Telosome, a mammalian telomere-associated complex formed by multiple telomeric proteins. $J$ Biol Chem 2004, 279(49):51338-51342.

11. Broccoli D, Chong L, Oelmann S, Fernald AA, Marziliano N, van Steensel B, Kipling D, Le Beau MM, de Lange T: Comparison of the human and mouse genes encoding the telomeric protein, TRF1: chromosomal localization, expression, and conserved protein domains. Hum Mol Genet 1997, 6(1):69-76.

12. Cooper JP, Nimmo ER, Allshire RC, Cech TR: Regulation of telomere length and function by a Myb-domain protein in fission yeast. Nature 1997, 385(6618):744-747.

13. Bilaud T, Koering CE, Binet-Brasselet E, Ancelin K, Pollice A, Gasser SM, Gilson E: The telobox, a Myb-related telomeric DNA binding motif found in proteins from yeast, plants and human. Nucleic Acids Res 1996, 24(7):1294-1303.

14. Vassetzky NS, Gaden F, Brun C, Gasser SM, Gilson E: Taz1p and Teb1p, two telobox proteins in Schizosaccharomyces pombe, recognize different telomere-related DNA sequences. Nucleic Acids Res 1999, 27(24):4687-4694 
15. Zhong Z, Shiue L, Kaplan S, de Lange T: A mammalian factor that binds telomeric TTAGGG repeats in vitro. Mol Cell Biol 1992, 12(11):4834-4843.

16. Smogorzewska A, de Lange T: Regulation of telomerase by telomeric proteins. Annu Rev Biochem 2004, 73:177-208

17. Lira CBB, Siqueira Neto JL, Khater L, Cagliari TC, Peroni LA, Reis JRR, Ramos CHI, Cano MIN: LaTBP1: a Leishmania amazonensis DNA-binding protein that associates in vivo with telomeres and GT-rich DNA using a myblike domain. Arch Biochem Biophys 2007, 465(2):399-409.

18. Broccoli D, Smogorzewska A, Chong L, de Lange T: Human telomeres contain two distinct Myb-related proteins, TRF1 and TRF2. Nature Gen 1997, 17(2):231-235.

19. Court R, Chapman L, Fairall L, Rhodes D: How the human telomeric proteins TRF1 and TRF2 recognize telomeric DNA: a view from highresolution crystal structures. EMBO Rep 2005, 6(1):39-45.

20. Opresko PL, von Kobbe C, Laine JP, Harrigan J, Hickson ID, Bohr VA: Telomere-binding protein TRF2 binds to and stimulates the Werner and Bloom syndrome helicases. J Bio/ Chem 2002, 277(43):41110-41119.

21. Takai H, Smogorzewska A, de Lange T: DNA damage foci at dysfunctional telomeres. Curr Biol 2003, 13(17):1549-1556

22. Celli GB, de Lange T: DNA processing is not required for ATM-mediated telomere damage response after TRF2 deletion. Nat Cell Biol 2005 7(7):712-718.

23. Lira CBB, Giardini MA, Siqueira Neto JL, Conte FF, Cano MIN: Telomere biology of trypanosomatids: beginning to answer some questions. Trends Parasitol 2007, 23(8):357-362.

24. Li B, Espinal A, Cross GAM: Trypanosome telomeres are protected by a homologue of mammalian TRF2. Mo/ Cell Biol 2005, 25(12):5011-5021.

25. Giardini MA, Lira CBB, Conte FF, Camillo LR, Siqueira Neto JL, Ramos CHI, Cano MIN: The putative telomerase reverse transcriptase component of Leishmania amazonensis: gene cloning and characterization. Parasitol Res 2006, 98(5):447-454

26. Stevens JR, Gibson W: The molecular evolution of trypanosomes. Parasitol Today 1999, 15(11):432-437.

27. Mao Z, Seluanov A, Jiang Y, Gorbunova V: TRF2 is required for repair of nontelomeric DNA double-strand breaks by homologous recombination. Proc Nat Acad Scienc USA 2007, 104(32):13068-13073.

28. Fotiadou $P$, Henegariu $O$, Sweasy JB: DNA Polymerase $B$ Interacts with TRF2 and induces telomere dysfunction in a murine mammary cell line. Cancer Res 2004, 64(11):3830-3837.

29. Munoz-Jordan JL, Cross GAM: Telomere shortening and cell cycle arrest in Trypanosoma brucei expressing human telomeric repeat factor TRF1. Mol Biochem Parasitol 2001, 114:169-181.

30. Fairall L, Chapman L, Moss H, de Lange T, Rhodes D: Structure of the TRFH dimerization domain of the human telomeric proteins TRF1 and TRF2. Mol Cell 2001, 8(2):351-361.

31. Cotrim PC, Paranhos GS, Mortara RA, Wanderley J, Rassi A, Camargo MA, da Silveira JF: Expression in Escherichia coli of a dominant immunogen of Trypanosoma cruzi recognized by human chagasic sera. J Clin Microbiol 1990, 28(3):519-524.

32. Neto JL, Lira CB, Giardini MA, Khater L, Perez AM, Peroni LA, dos Reis JR, Freitas-Junior $\mathrm{LH}$, Ramos $\mathrm{CH}$, Cano Ml: Leishmania replication protein A-1 binds in vivo single-stranded telomeric DNA. Biochem Biophys Res ommun 2007, 358(2):417-23.

33. Forsythe GR, McCulloch R, Hammarton TC: Hydroxyurea-induced synchronisation of bloodstream stage Trypanosoma brucei. Mol Biochem Parasitol 2009, 164(2):131-136.

doi: $10.1186 / 1471-2180-10-136$

Cite this article as: da Silva et al., The Leishmania amazonensis TRF (TTAGGG repeat-binding factor) homologue binds and co-localizes with telomeres BMC Microbiology 2010, 10:136

\section{Submit your next manuscript to BioMed Centra} and take full advantage of:

- Convenient online submission

- Thorough peer review

- No space constraints or color figure charges

- Immediate publication on acceptance

- Inclusion in PubMed, CAS, Scopus and Google Scholar

- Research which is freely available for redistribution

Submit your manuscript at www.biomedcentral.com/submit
C Biomed Central 